\title{
COMPENSAÇÃO DE EMISSÕES DE CO 2 DURANTE A 14a SEMANA NACIONAL DE CIÊNCIA E TECNOLOGIA NO VALE DO PARAÍBA-SP
}

\author{
$\mathrm{CO}_{2}$ EMISSION COMPENSATION DURING THE $14^{\text {th }}$ NATIONAL WEEK OF SCIENCE AND \\ TECHNOLOGY IN THE PARAÍBA-SP VALLEY
}

\author{
Humberto Gallo Junior ${ }^{1}$, Alcinéa Guimarães de Castro², Patrícia Mie Matsuo ${ }^{3}$, Débora Olivato ${ }^{4}$, \\ Douglas Reis Souza ${ }^{5}$, Raquel Trajber ${ }^{6}$, Getúlio Martins ${ }^{7}$, Carolina Tosetto ${ }^{8}$ \\ ${ }^{1,2}$ Instituto Florestal/SMA-SP, Taubaté, São Paulo, Brasil-humbertogallojr@gmail.com \& \\ neia.gcastro@ig.com.br \\ 3, 4,6,8 Cemaden/MCTI, São José dos Campos, São Paulo, Brasil - patricia.matsuo@cemaden.gov.br, \\ debora.olivato@gmail.com, rachel.trajber@cemaden.gov.br \& carolina.pimentel@cemaden.gov.br \\ 5, 7 Faculdade de Roseira, Roseira, São Paulo, Brasil-douglas.reis@faroroseira.edu.br \& \\ getulio.martins@faroroseira.edu.br
}

\begin{abstract}
RESUMO
Atualmente, ações humanas intensificaram as emissões de Gases de Efeito Estufa (GEE), decorrentes sobretudo, da queima de combustíveis fósseis, do desmatamento, do uso e manejo inadequado do solo. Este trabalho teve por objetivo realizar o cálculo para compensação ambiental dos GEE emitidos na 14a edição da "Semana Nacional de Ciência e Tecnologia (SNCT) no Vale do Paraíba, realizada nos dias 26 e 27 de outubro de 2017 em São José dos Campos-SP. $O$ evento contou com a participação de mais de 300 estudantes de quinze escolas públicas parceiras do projeto Cemaden Educação e PROEX da UNESP/SJC. Foi realizado o cálculo das emissões de carbono por meio da ferramenta disponibilizada pelo Programa GHG Protocol - GVCES da Fundação Getúlio Vargas, conforme diretrizes do Painel Intergovernamental de Mudanças Climáticas - IPCC/ONU. Para tanto, foram analisados os seguintes critérios: emissões diretas (combustão móvel); emissões fugitivas (equipamento de refrigeração e de incêndio); eletricidade comprada; emissões indiretas (resíduos sólidos, efluentes, consumo de água, refeições, e material de consumo e divulgação). Como resultado foi contabilizada a emissão total de $11,34 \mathrm{t} \mathrm{CO}_{2}$, estabelecendo-se como compensação o plantio de 72 mudas nativas do Bioma Mata Atlântica. As mudas foram produzidas e plantadas no Viveiro Florestal de Taubaté, com o envolvimento de estudantes da região e parceiros organizadores da 14a SNCT.
\end{abstract}

PALAVRAS-CHAVE: Aquecimento global, Compensação de carbono, Mudanças climáticas.

\section{ABSTRACT}

Currently, human actions have intensified greenhouse gas (GHG) emissions, mainly due to the burning of fossil fuels, deforestation, use and inadequate management of the soil. The objective of this work was to calculate the environmental compensation of GHGs issued in the 14th edition of the National Science and Technology Week (SNCT) in Vale do Paraíba, held from October 26 to 27, 2017 in São José dos Campos, SP. The event was attended by more than 300 students, from fifteen public schools partners of the project Cemaden Education and PROEX of UNESP / SJC. The carbon offset study was developed through the tool provided by the GHG Protocol - GVCES Program of the Getúlio Vargas Foundation, according to guidelines of the Intergovernmental Panel on Climate Change - IPCC / ONU. For that, the following criteria were analyzed: direct emissions (mobile combustion); fugitive emissions (cooling and fire equipment); electricity purchased; indirect emissions (solid waste, effluents, water consumption, meals, and consumables and dissemination). As a result, the total emission of $11.34 \mathrm{tCO}_{2}$ was recorded, establishing as compensation the planting of 72 native seedlings of the Atlantic Forest Biome. The seedlings were produced and planted in the Forest Nursery of Taubaté, with the involvement of students from the region and partners organizing the 14th SNCT.

KEYWORDS: Global warming, Carbon offset, Climate changes. 


\section{INTRODUÇÃO}

Segundo o Painel Intergovernamental de Mudanças Climáticas (IPCC, 2013), existem evidências científicas de que as mudanças climáticas estão relacionadas com as atividades antrópicas, devido principalmente ao consumo de combustíveis fósseis (carvão mineral, petróleo e gás natural), assim como pelos desmatamentos e queimadas.

O relatório de avaliação das concentrações dos Gases de Efeito Estufa (GEE) de 2013 concluiu que a atmosfera e o oceano aqueceram, a quantidade de gelo e neve diminuiu, o nível do mar se elevou e as concentrações de GEE aumentaram nas últimas décadas. De acordo com o relatório, foi confirmado o aumento das concentrações do $\mathrm{CO}_{2}$, chegando a 400 partes por milhão (ppm) no ano de 2013, quase o dobro do valor da época pré-industrial que correspondia a 277 ppm (IPCC, 2013).

$\mathrm{O} \mathrm{CO}_{2}$ é o gás mais emitido para atmosfera e é apontado como o responsável por $80 \%$ do aquecimento global, sendo que o desmatamento contribui com $25 \%$ dessas emissões. No Brasil, além dos desmatamentos, as emissões estão associadas, também, às queimadas de florestas (MCT, 2014). Matthews et al. (2014) destacou que o país ocupa um dos primeiros lugares no ranking das emissões desse gás. Dados recentes do Sistema de Estimativas de Emissões de Gases de Efeito Estufa, do Observatório do Clima (2017) apontam que o Brasil emitiu 2,091 bilhões de toneladas de $\mathrm{CO}_{2}$ no ano de 2015 e 2,278 bilhões de toneladas em 2016, o que corresponde a 3,4\% do total mundial.

Ao avaliar as emissões de $\mathrm{CO}_{2}$ associadas ao consumo de energia no Paraná, Sanquetta et al. (2017) destacaram a grande elevação de emissões também neste setor, salientando a necessidade de ampliar a participação de energias renováveis na geração de energia elétrica no Brasil.

Ao longo dos últimos anos, também vem sendo discutidas formas de compensação das emissões dos GEE e da mitigação dos efeitos das mudanças climáticas, tais como: o aumento da cobertura florestal (reflorestamento), para fixação do carbono atmosférico e a geração de energia elétrica, utilizando fontes renováveis.

O conceito de sequestro de carbono foi consagrado pela Conferência de Quioto, no ano de 1997, com o objetivo de conter e reverter o acúmulo de $\mathrm{CO}_{2}$ na atmosfera e com a intenção de minimizar os impactos ambientais, referentes às mudanças climáticas quanto ao aquecimento global e a elevação dos gases de efeito estufa.
O sequestro de carbono refere-se à retirada do gás carbono atmosférico e segundo Renner (2004) pode ser definido como a capacidade fotossintética que os vegetais possuem de fixar o $\mathrm{CO}_{2}$ da atmosfera, no qual é sintetizado através da luz na forma de carboidratos que são depositados na parede celular do vegetal.

Barbosa (2013) relaciona algumas ações que contribuem para a redução do $\mathrm{CO}_{2}$ na atmosfera, como: a conservação de estoques de carbono nos solos, nas florestas e em outras formações; a preservação de florestas nativas; a implantação de florestas e de sistemas agroflorestais e; a recuperação de áreas degradadas.

O estabelecimento de novas florestas, os reflorestamentos, como sumidouros de carbono e os desflorestamentos (supressão das florestas) devem ser computados para o atendimento das metas de redução de cada país desenvolvido (MARTINS, 2004).

O Brasil se comprometeu na Convenção Quadro das Nações Unidas Sobre Mudanças Climáticas, em sua 21a Conferência das Partes (COP21) realizada em Paris, no ano de 2015 , em reduzir os GEE a $37 \%$ abaixo dos níveis de 2005, até o ano de 2025 e uma redução subsequente de $47 \%$ até 2030 . Para isso, o país compromete-se com um aumento de $18 \%$ na participação de bioenergia sustentável e com a estimada de $45 \%$ de energias renováveis na composição de sua matriz energética, até 2030 e, também, restaurar e reflorestar 12 milhões de hectares de áreas de florestas.

A região do Vale do Paraíba conta com mais de três séculos de ocupação, acumulando em sua paisagem as marcas resultantes das combinações dos diferentes ciclos econômicos (açúcar, café, gado, eucalipto e indústria), ocorridos nos diversos momentos de sua história. De acordo com Kronka et al. (2005), o maior índice de desmatamento na região, assim como em todo o estado de São Paulo, ocorreu entre as décadas de 1960 e 1970. Pesquisa recente desenvolvida pela EMBRAPA (RONQUIM et al., 2016) concluiu que as áreas de floresta passaram de 250 mil para 455 mil hectares entre os anos de 1985 e 2015, com aumento de $83 \%$ em floresta nativa na porção paulista do Vale do Paraíba, correspondendo atualmente a $33 \%$ da área. No entanto, o trabalho aponta que o aumento das áreas de florestas ocorreu principalmente por regeneração natural em áreas com restrições para a agricultura e pecuária e não pelo plantio de novas árvores.

Nos dias 26 e 27 de outubro de 2017 aconteceu em São José dos Campos-SP, a Semana Nacional de Ciência e Tecnologia no Vale do Paraíba (http://www.cemaden.gov.br/snct2017/), organizada 
pelo Centro Nacional de Monitoramento e Alertas de Desastres Naturais (CEMADEN), Instituto de Pesquisas Espaciais (INPE) e Universidade Estadual Paulista (UNESP) São José dos Campos. O objetivo do evento foi oferecer às escolas da região e ao público em geral oportunidades de interação com cientistas, participação em oficinas, palestras e visitas a laboratórios.

O presente trabalho teve como objetivo estabelecer a compensação das emissões de $\mathrm{CO}_{2}$ decorrentes desse evento por meio da produção e plantio de mudas de espécies nativas, aliada a ações de educação ambiental e educação para redução de riscos de desastres.

\section{MATERIAL E MÉTODOS}

\section{Área de Estudo}

O Viveiro Florestal de Taubaté foi criado em 1960 através do Decreto Estadual 36.771, possuindo uma área de 10 ha, recobertos por vegetação em estágio avançado de sucessão, vegetação secundária e campo antrópico (Figura 1).

O Viveiro é administrado pelo Instituto Florestal, da Secretaria de Meio Ambiente do Estado de São Paulo, possuindo infraestrutura com sede administrativa, centro de visitantes, laboratório de pesquisa e áreas para atividades de educação ambiental.

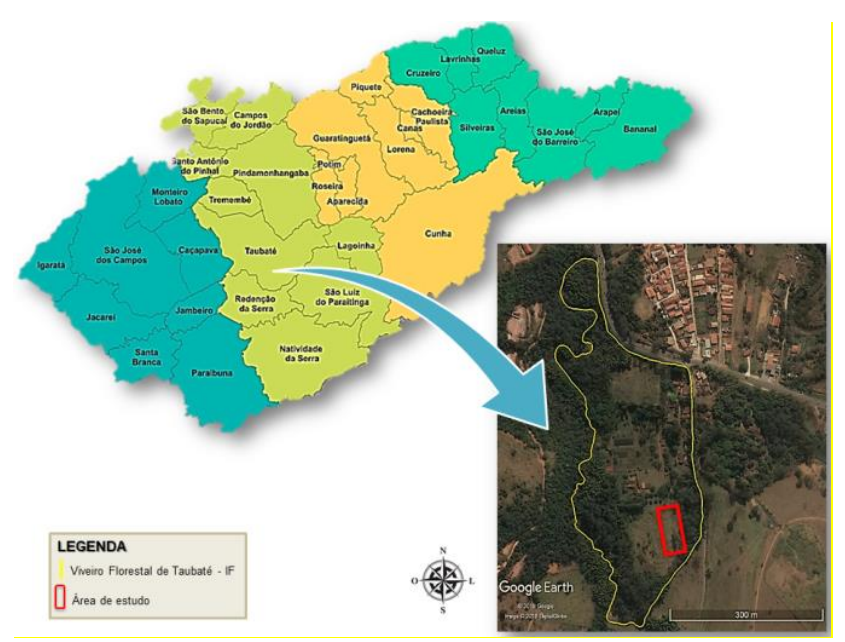

Figura 1. Localização da área de estudo no Viveiro Florestal de Taubaté. Fonte: EMPLASA - UDI, 2016. Elaboração: Douglas Reis Souza.

Localiza-se no bairro do Registro, município de Taubaté-SP, na foz do Ribeirão das Antas. A microbacia hidrográfica do Ribeirão das Antas está situada em região de ocorrência da Mata Atlântica, cujo ecossistema original se caracteriza como Floresta Ombrófila Densa (Floresta
Pluvial Tropical ou Floresta Sempre Verde). A geologia é caracterizada por terrenos cuja formação litológica predominante é de rochas cristalinas com idade précambriana englobando as diferentes unidades compostas de rochas ígneas e metamórficas (SIMÕES, 2011).

O clima, pela classificação de Koeppen, é do tipo Cwa, estacional com verão quente com chuvas intensas e inverno marcado por frio e período de seca. A temperatura média dos meses de verão é superior a $22{ }^{\circ} \mathrm{C}$ enquanto a do mês mais frio é inferior a 18 으. A precipitação anual é de $1335 \mathrm{~mm}$, com 42\% deste valor concentrando-se de dezembro a fevereiro, meses que apresentam precipitações que excede a $200 \mathrm{~mm}$ (FISCH, 1995). Os solos da região da bacia do Una são formados, principalmente, por latossolos vermelho-amarelos, considerados porosos e permeáveis, facilitando a recarga dos aquíferos da região (SANTORO et al., 2007).

Batista \& Batista (2009) avaliaram o uso e ocupação do solo na bacia do Ribeirão das Antas e correlacionaram as informações com as Áreas de Preservação Permanente (APP). Os autores verificaram a sobreposição entre os usos e áreas de APP: as pastagens ocupam 866 ha de topo de morro, 415 ha em margens de rio e 121 ha em nascentes; os reflorestamentos ocupam 49 ha de topos de morro, 8 ha em margens de rio e 4 ha em nascentes. Desta forma, concluíram que a maior parte da bacia está em desconformidade com o Código Florestal Brasileiro. Segundo dados do Inventário Florestal de São Paulo (SÃO PAULO, 2005), o município de Taubaté possuía em 2005 apenas $9,84 \%$ de seu território recoberto por vegetação natural, sendo $3,44 \%$ de mata, $6,39 \%$ de capoeira e 0,01 de vegetação de várzea.

\section{Cálculo das emissões de $\mathrm{CO}_{2}$ na SNCT do Vale do Paraíba}

A 14ạ edição da SNCT do Vale do Paraíba contou com a participação de mais de 300 estudantes, de quinze escolas públicas parceiras do projeto Cemaden Educação e PROEX - UNESP SJC. Estas escolas pertencem aos seguintes municípios paulistas: Caçapava; Cunha; Campos do Jordão; São Luiz do Paraitinga; Vargem Grande Paulista; Paraibuna; São Paulo; Lorena; Ubatuba e São José dos Campos (Quadro I).

As atividades compreenderam palestras, feira de ciências, oficinas, visitas em instituições de pesquisa e de ensino. No dia 26 de outubro as atividades ocorreram na UNESP campus São José dos Campos e no Parque Vicentina Aranha (Figura 2). No dia 27 de outubro foram realizadas Oficinas no Parque Tecnológico de São José dos Campos, oferecidas pelo Cemaden, INPE e Unesp. 
Quadro I. Relação das escolas que participaram da SNCT 2017 no Vale do Paraíba.

\begin{tabular}{|c|c|c|c|c|}
\hline Data & Escolas & $\begin{array}{l}\text { Quantidade de } \\
\text { alunose } \\
\text { professores }\end{array}$ & Municipio & $\begin{array}{c}\text { Quilometragem } \\
\text { aproximada (ida e volta) }\end{array}$ \\
\hline \multirow{11}{*}{ 26/out } & EE Paulo Virgínio & 45 & Cunha & $270 \mathrm{~km}$ \\
\hline & EE Monsenhor I. Goia & 15 & Săo Luis do Paraitinga & $195 \mathrm{~km}$ \\
\hline & EE Dr Pereira Mattos & 50 & Caçapava & $54 \mathrm{~km}$ \\
\hline & EE Profa. Ma. Ap. F.B. Araujo & 10 & Caçapava & $64 \mathrm{~km}$ \\
\hline & EE Ilza IC Coppio & 30 & São José dos Campos & $20 \mathrm{~km}$ \\
\hline & EM Vera Babo & 15 & São José dos Campos & $25 \mathrm{~km}$ \\
\hline & EE Geraldo M dos Santos & 15 & Paraibuna & $80 \mathrm{~km}$ \\
\hline & $\begin{array}{l}\text { EM Padre João Renaudin } \\
\text { Ranville }\end{array}$ & 15 & Lorena & $130 \mathrm{~km}$ \\
\hline & EE Exp. C. Freire e EE Theodoro & 15 & Campos do Jordão & $200 \mathrm{~km}$ \\
\hline & EE Santos Dias Silva & 15 & Săo Paulo & $250 \mathrm{~km}$ \\
\hline & EE Florentina & 3 & Ubatuba & $280 \mathrm{~km}$ \\
\hline \multirow{5}{*}{ 27/out } & EE Paulo Virgínio & 15 & Cunha & $270 \mathrm{~km}$ \\
\hline & EE Dr Pereira Mattos & 45 & Caçapava & $54 \mathrm{~km}$ \\
\hline & EE Alexandre Fleming & 15 & Vargem Grande do Sul & $600 \mathrm{~km}$ \\
\hline & EE Monsenhor I Goia & 32 & São José dos Campos & $25 \mathrm{~km}$ \\
\hline & EE Profa. Ma. Ap. F.B. Araujo & 15 & Caçapava & $64 \mathrm{~km}$ \\
\hline TOTAL & & 350 & & $2581 \mathrm{~km}$ \\
\hline
\end{tabular}
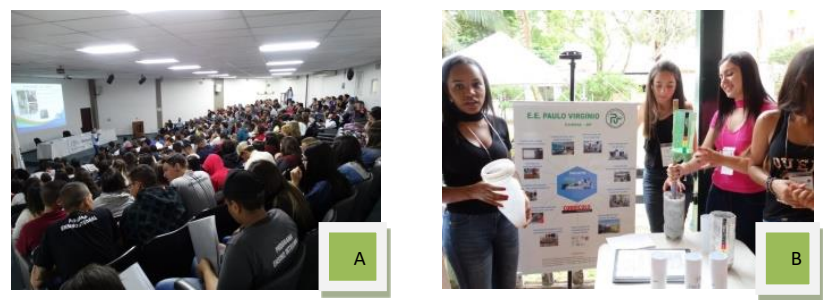

Figura 2. Atividades desenvolvidas durante a 14a SNCT do Vale do Paraíba. A: Palestra. B: Exposição de trabalhos. Fonte: Ascom - Cemaden - 2017.

A ferramenta de cálculo abrangeu os três escopos definidos pelo programa e todas as potenciais fontes de emissão previstas pelo IPCC (GREENHOUSE GAS PROTOCOL, 2018).

Para tal, foram analisados os seguintes critérios: emissões diretas (combustão móvel); emissões fugitivas (equipamento de refrigeração e de incêndio); eletricidade comprada; emissões indiretas (resíduos sólidos, efluentes, consumo de água, refeições, e material de consumo e divulgação).

Para o cálculo das emissões foi utilizada a ferramenta disponibilizada pelo Programa GHG Protocol - GVCES da Fundação Getúlio Vargas, Versão 3.1 - 2017, conforme diretrizes do Painel Intergovernamental de Mudanças Climáticas - IPCC da ONU. Esta ferramenta visa auxiliar os gestores públicos e privados no processo de elaboração dos inventários de GEE e está disponível no site da FGV.

A compensação considerou a Resolução SMA 30 de $15 / 05 / 2009$, que orienta projetos voluntários de reflorestamento para compensação de emissões de gases de efeito estufa, particularmente no que se refere ao plantio de espécies nativas de ocorrência regional, com potencial de regeneração natural, em área próxima ao curso d'água.

\section{Produção de mudas para compensação ambiental}

Em relação à produção das mudas, inicialmente foi construída uma sementeira (áreas onde são semeadas, principalmente propágulos de espécies que demoram em germinar), sendo subdivida em espaços para a germinação isolada de cada espécie.

As espécies utilizadas no plantio foram definidas de acordo com as diretrizes contidas no artigo 6 da Resolução SMA 8 de 7/3/2007, priorizando-se espécies florestais nativas de ocorrência regional.

Após efetuar a limpeza do local, foi acrescentada a mistura do substrato (a fim de proporcionar boa drenagem e ser isento de micro-organismos patogênicos) e areia, nivelando a área para melhor drenagem.

Posteriormente foi feita a semeadura em cada respectivo espaço, sendo efetuada a rega duas vezes ao dia (de manhã e à tarde), de forma a evitar o aparecimento de doenças e o encharcamento dos canteiros.

$\mathrm{Na}$ etapa seguinte, foi realizado o enchimento manual dos recipientes (sacos plásticos de polietileno preto) com a mistura do substrato preparado. Os recipientes escolhidos não ficam totalmente cheios, deixando-se de 1 $\mathrm{cm}$ a $2 \mathrm{~cm}$ livres na superfície para que possa ser retida mais água no momento da irrigação.

Foi realizado o acompanhamento diário do desenvolvimento da germinação, por estágios. Quando as mudas atingiram altura de 3 a $7 \mathrm{~cm}$ (em geral apresentando dois pares de folhas, dependendo da espécie) foi efetuado o processo de repicagem para os recipientes.

A etapa final da produção foi a formação de canteiros, com o enfileiramento dos recipientes nas mesas, tendo em vista que recipientes devem ser colocados formando linhas e colunas. É importante ressaltar que esse enfileiramento não deve ultrapassar a largura máxima de $1 \mathrm{~m}$, para não dificultar tratos culturais nas mudas centrais, como irrigação, luminosidade e controle de pragas e doenças.

Algumas medidas foram tomadas antes do plantio, como a identificação e remoção de fatores de degradação, tais como espécies vegetais invasoras e exóticas, especialmente as gramíneas agressivas (capim colonião - Panicum maximum, braquiária - Brachiaria brizantha, entre outras). Os berços foram abertos nas dimensões mínimas de $40 \mathrm{~cm}$ (largura) x $40 \mathrm{~cm}$ (comprimento) $\times 40 \mathrm{~cm}$ (profundidade). A terra retirada foi homogeneizada com substrato/condicionador para melhor qualidade, melhoria física e mecânica do solo, 
favorecendo sua descompactação.

A área selecionada para o plantio encontra-se nas proximidades do Ribeirão das Antas, afluente da bacia do Rio Una, entre as coordenadas geográficas $23^{\circ} 08^{\prime} 10^{\prime \prime} \mathrm{e}$ 23 $08^{\prime} 26^{\prime \prime}$ de latitude Sul e $45^{\circ} 30^{\prime} 11^{\prime \prime}$ e $45^{\circ} 30^{\prime} 23^{\prime \prime}$ de longitude $W$, com altitude de $560 \mathrm{~m}$. Possui $602 \mathrm{~m}^{2}$ e não apresenta vestígios de pastoreio, sendo caracterizada como campo antrópico.

Para a realização do plantio, foi adotado o espaçamento de $3 \mathrm{~m} \times 2 \mathrm{~m}$ entre as mudas, sendo colocadas estacas para facilitar a identificação (Figura 3 ).

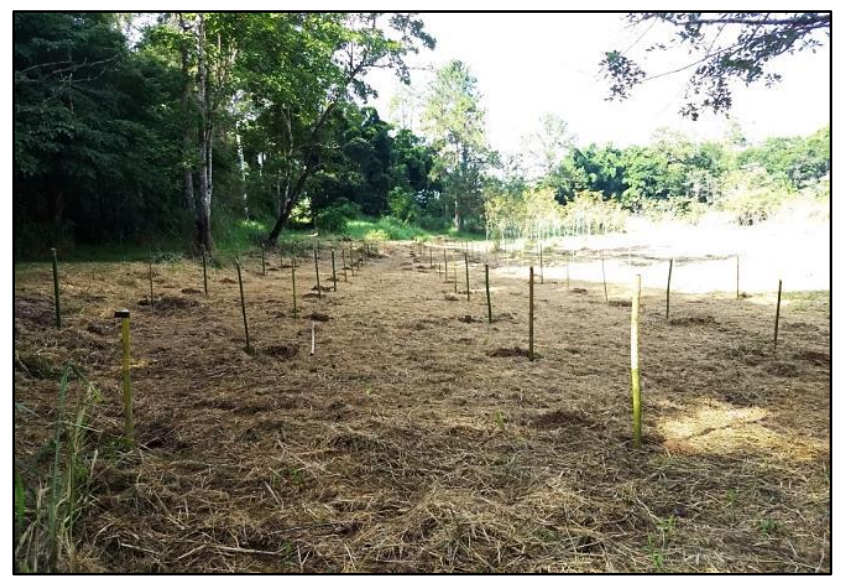

Figura 3. Preparação da área para o plantio.

\section{RESULTADOS E DISCUSSÃO}

\section{Cálculo das emissões}

Para determinação da quantidade de árvores nativas a serem plantadas para sequestro dos GEE emitidos à atmosfera durante o evento, foram utilizados os dados apresentados no Quadro II.

O Quadro III apresenta o cálculo das emissões de $\mathrm{CO}_{2}$ com as atividades realizadas durante a 14a SNCT e definição da compensação por meio do plantio de espécies nativas.

A definição da combustão móvel teve como base a distância percorrida pelo público, sendo alunos e professores de escolas de diversos municípios, conforme Quadro I (2581 km), além do trajeto dos demais participantes, considerando-se o deslocamento de $20 \mathrm{~km}$ para 50 pessoas $=1000 \mathrm{~km}$.

Os dados referentes aos resíduos sólidos, efluentes e consumo de água foram estimados pela comissão organizadora da SNCT.
Quadro II. Dados utilizados para o cálculo de emissões de $\mathrm{CO}_{2}$ durante a 14 a $\mathrm{SNCT}$.

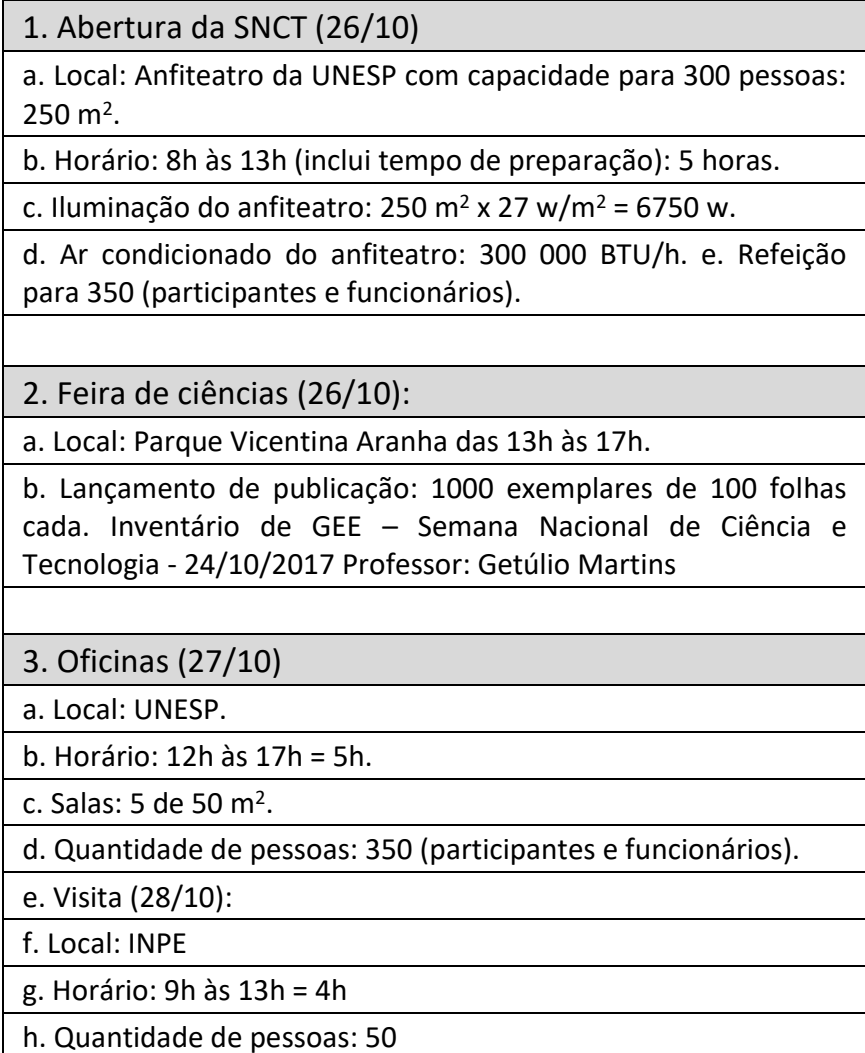

Quadro III. Cálculo das emissões de $\mathrm{CO}_{2}$ na 14 a SNCT e definição da compensação ambiental.

\section{Escopo 1 (emissões diretas)}

a. Combustão móvel: distância percorrida = $3581 \mathrm{~km}$

b. Emissões fugitivas: ar condicionado, equipamentos de refrigeração e extintores de incêndio $=32 \mathrm{~kg} 2$.

2. Escopo 2 (eletricidade comprada)

a. Iluminação, ar condicionado e refrigeração $=219$ kwh

3. Escopo 3 (emissões indiretas)

a. Resíduos sólidos: 0,70 toneladas

b. Efluentes: $67,20 \mathrm{~m}^{3}$

c. Consumo de água fornecida pela Sabesp: $84 \mathrm{~m}^{3}$

d. Refeição de participantes e funcionários: 700 unidades

e. Papel e material de divulgação: $1,25 \mathrm{~m}^{2}$

Cálculo da compensação

1. Emissão total $=11,34 \mathrm{t} \mathrm{CO}_{2}$

2. Quantidade de árvores a serem plantadas: $\mathbf{7 2}$ árvores.

Estimou-se a geração de resíduos por alunos e professores, sendo $350 \times 1 \mathrm{~kg} /$ pessoas/dia $\times 2$ dias $=700$ $\mathrm{kg}=0,70 \mathrm{t}$. $\mathrm{O}$ valor per capita considerou a geração de resíduos na preparação das refeições, embalagens, restos, 
incluindo resíduos secos e úmidos, sem separação. Foi estimado o consumo da água fornecida pela Sabesp, sendo $350 \times 120$ litros / pessoa / dia $\times 2$ dias $=84 \mathrm{~m}^{3}$. 0 valor per capita considerou água para consumo, limpeza e preparo de refeições. A estimativa de emissão de gases de efeito estufa foi feita considerando $0,061 \mathrm{~kg} \mathrm{CO} / 2 \mathrm{~m}^{3}$ de acordo com dados obtidos na SAAEG - Guaratinguetá, uma vez que a Sabesp não disponibiliza essa informação. Quanto aos efluentes, foi considerado o retorno como esgoto, de $80 \%$ da água consumida $=84 \times 0,80=67,20 \mathrm{~m}^{3}$.

A quantidade de árvores foi estimada de acordo com a recomendação da Secretaria de Meio Ambiente do Estado de São Paulo por meio da Resolução SMA 30 de 2009, que considera estoque máximo de $350 \mathrm{t} \mathrm{CO}_{2}$ / ha em projetos de compensação. Foi considerada a absorção de $190 \mathrm{~kg}$ de $\mathrm{CO}_{2} /$ árvore, sendo 11,34 t CO $2 / 0,19 \mathrm{t} \mathrm{CO}_{2}=60$ árvores. Considerando um acréscimo de $20 \%$ devido às imprecisões nas estimativas e perdas eventuais, chegouse ao total de 72 árvores.

\section{Plantio de mudas para compensação ambiental.}

A ação de plantio ocorreu no dia 21 de março de 2018, em evento realizado através da parceria entre o Instituto Florestal e o Centro Nacional de Monitoramento e Alertas de Desastres Naturais (CEMADEN) (Figura 3). O referido evento serviu como celebração ao Dia Internacional das Florestas e da Semana Mundial da Água e contou com palestras sobre a importância da preservação e recuperação das matas ciliares para evitar problemas de erosão e assoreamento dos rios e prevenir riscos de desastres relacionados às inundações. Também foi abordado o processo de produção de mudas e os procedimentos adequados para a realização da ação de recuperação ambiental.

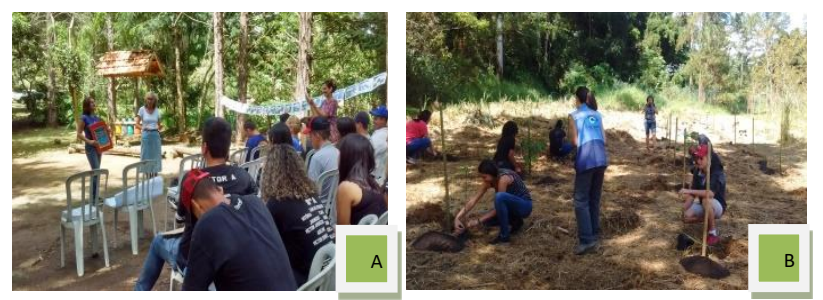

Figura 3. Atividade de plantio de mudas no Viveiro Florestal de Taubaté. A: Palestra: B: Plantio com participação de alunos da Escola Estadual José Mazella.

A atividade foi realizada em área do Viveiro Florestal de Taubaté, com a participação dos alunos de ensino médio da Escola Estadual José Mazella, localizada nas proximidades da área a ser recuperada. A perspectiva foi a de estimular o envolvimento de estudantes moradores da região, possibilitar a compreensão sobre a importância da conservação e recuperação das áreas de preservação permanente para a redução de riscos de desastres e da melhoria da qualidade ambiental e de vida das comunidades.

A Tabela 1 apresenta as espécies utilizadas na compensação e quantidades de mudas plantadas. Será feito o acompanhamento e monitoramento semanal do desenvolvimento das mudas por um prazo de 2 anos. Será feito o manejo da área para assegurar o desenvolvimento das mudas, com a utilização das técnicas de roçada, coroamento, combate a formigas cortadeiras e adubação de cobertura. Serão coletados dados de variáveis dendrométricas como altura e diâmetro do coleto.

Tabela 1. Relação das mudas plantadas para compensação das emissões de $\mathrm{CO}_{2}$ na SNCT 2017.

\begin{tabular}{ccc}
\hline Nome Popular & Nome Científico & Quantidade \\
\hline angico-vermelho & Paraptadenia rigida & 3 \\
araçá & Psidium cattleianum & 3 \\
embira de sapo & Lonchocarpus guilleminianus & 3 \\
guauvira & Patagonula americana & 4 \\
guarantã & Esenbeckia leiocarpa & 4 \\
ingá & Inga sessilis & 4 \\
ipê rosa & Tabebuia pentaphylla & 4 \\
ipê roxo & Tabebuia impetiginosa & 2 \\
jatobá & Hymenaea courbaril & 2 \\
jequitibá & Cariniana legalis & 3 \\
\hline leiteiro & Tabernaemontana hystrix & 5 \\
manacá da serra & Tibouchina mutabilis & 3 \\
mulungu & Erythrina speciosa & 5 \\
\hline olho de cabra & Ormosia arborea & 2 \\
paineira & Seiba speciosa & 2 \\
pau jangada & Heliocarpus popayanensis & 5 \\
pau viola & Cytharexyllum myrianthum & 3 \\
\hline peroba & Aspidosperma polyneuron & 3 \\
\hline quaresmeira & Tibouchina granulosa & 4 \\
sibipiruna & Caesalpinia peltophoroides & 4 \\
\hline sobrasil & Colubrina glandulosa & 4 \\
\hline Total & 21 espécies & $\mathbf{7 2 ~ i n d i v i ́ d u o s ~}$ \\
\hline & & \\
\hline & & \\
\hline
\end{tabular}

\section{Discussão e perspectivas}

A utilização das plantas na absorção de $\mathrm{CO}_{2}$ pelo processo de fotossíntese está contemplada como forma de estabilizar as concentrações dos GEE emitidos na atmosfera pela Convenção-Quadro das Nações Unidas sobre Mudanças Climáticas (UNFCCC, sigla em inglês United Nations Framework Convention on Climate 
Change), tratado internacional resultante da Conferência das Nações Unidas para o Meio Ambiente e o Desenvolvimento, no ano de 1992, firmado por quase todos os países do mundo.

Os projetos de restauração ecológica em florestas tropicais tem se pautado no plantio de espécies vegetais nativas, com o objetivo de favorecer os processos ecológicos e a dinâmica florestal, buscando a reconstituição do ambiente florestal, na tentativa de reparar danos provocados pelo homem nesses ecossistemas e mais recentemente para computação de créditos de carbono.

Neste contexto, é fundamental a realização de projetos que contemplem ações de restauração ecológica e educação ambiental como forma de ampliar a compreensão sobre as mudanças climáticas e os riscos socioambientais e estimular práticas de prevenção, adaptação e mitigação.

Conforme o estudo desenvolvido pela Embrapa (RONQUIM et al., 2016) a recuperação de 205 mil hectares de florestas nativas na região do Vale do Paraíba, entre 1985 e 2015, representou o sequestro de 35,4 milhões de toneladas de $\mathrm{CO}_{2}$. Este é um dado bastante significativo e que demonstra a importância da restauração florestal para a redução da concentração de $\mathrm{CO}_{2}$ na atmosfera. A região apresenta extensas áreas passíveis de desenvolvimento de projetos de restauração ecológica, apresentando, portanto, um grande potencial para sequestro de $\mathrm{CO}_{2}$, contribuindo desta forma para o cumprimento das metas nacionais.

Diversos pesquisadores tem se dedicado ao estudo da absorção de $\mathrm{CO}_{2}$ por meio de reflorestamentos. Melo \& Durigan (2006) verificaram que plantios com espécies nativas possibilitaram o incremento médio de $5,2 \mathrm{tC} \mathrm{ha}^{-1}$ $a_{n o}^{-1}$ na região Do Vale do Paranapanema, São Paulo. Preiskorn (2011) obteve uma taxa média de fixação de carbono de 4,6 tC ha-1 ano-1 para plantios de restauração em municípios do interior do São Paulo. Miranda (2008) estudou plantios de restauração em áreas de Floresta Estacional Semidecidual na região sudoeste do estado de São Paulo, obtendo valores de incremento médio de carbono entre 4,8 e 5,6 $\mathrm{t} \mathrm{ha}^{-1} \mathrm{ano}^{-1}$

Santos et al. (2010) constataram que seria necessário o plantio de 1702 mudas nativas do Cerradão em 1,02 ha para neutralizar $218 \mathrm{tCO}_{2 \text { eq. }}$ geradas no campus da Universidade Federal de Tocantins

Brianezi et al. (2014) calcularam a emissão total de $6.034,18$ tCO $_{2 \text { eq. }}$ para o campus da Universidade Federal de Viçosa entre outubro de 2010 e outubro de 2011, com um total de 5.307,16 $\mathrm{tCO}_{2 \text { eq. }}$ de sumidouros para o mesmo período, sendo $43,31 \%$ deste valor referente às áreas de reflorestamento. Estes autores salientam que, além do montante de emissões, as características da vegetação, local e plano de manejo adotado também podem alterar os resultados obtidos.

Duarte \& Marchetto (2014) calcularam o total de emissões de 740,035 toneladas de $\mathrm{CO}_{2}$ para a Feira do Empreendedor - MT de 2011, estabelecendo como compensação o plantio de 4.112 árvores.

A utilização da metodologia desenvolvida pelo programa GHG Protocol - GVCES da Fundação Getúlio Vargas, foi indispensável para o sucesso da resultante do cálculo de $\mathrm{CO}_{2}$ emitido durante a 14a SNTC do Vale do Paraíba. Com isso, consolidou-se a ideia da compensação dos GEE por meio da reposição florestal. Durante a realização do plantio para compensação das emissões, verificou-se o grande interesse dos alunos de ensino médio sobre o tema e a disposição para participar das atividades, o que demonstra o potencial para continuidade do projeto.

A continuidade do trabalho compreenderá o acompanhamento e monitoramento do desenvolvimento das mudas com a participação dos alunos da Escola Estadual José Mazella. A partir da equação alométrica desenvolvida por Higuchi et al. (1998) e utilizada por Castro (2017) para os levantamentos da tese de doutoramento realizados em São Luiz do Paraitinga-SP, pretende-se estimar futuramente $\mathrm{o} \mathrm{CO}_{2}$ capturado pelas árvores plantadas. Esta metodologia relaciona as dimensões de diâmetro à altura do peito (DAP) e altura $(\mathrm{H})$ da árvore para estimativa da biomassa e cálculo dos valores de Peso Fresco (PF-biomassa), Peso Seco (PS), o Carbono ( $C$ em $\mathrm{Kg}$ ) e Carbono (C tonelada) para cada espécie. Também será trabalhada com os alunos a importância da conservação e restauração de áreas de preservação permanente para prevenção de riscos socioambientais, mitigação dos efeitos das mudanças climáticas e melhoria da qualidade dos recursos hídricos.

\section{CONSIDERAÇÕES FINAIS}

A Semana Nacional de Ciência e Tecnologia é uma iniciativa de grande relevância no contexto nacional, pois possibilita a integração de ações de diversas instituições e a disseminação da ciência junto aos alunos dos diversos níveis e também do público em geral. A realização do cálculo das emissões de $\mathrm{CO}_{2}$ e compensação para este evento na região do Vale do Paraíba representou o esforço para unir conhecimento científico, educação e práticas sustentáveis. A perspectiva é de realizar o cálculo 
das emissões para os eventos futuros e ampliar a participação de alunos de escolas públicas da região nesta dinâmica, utilizando-se estratégias ativas de ensinoaprendizagem para proporcionar o aumento do conhecimento sobre a temática e estimular o plantio de espécies nativas.

\section{AGRADECIMENTOS}

Aos organizadores da 14a Semana Nacional de Ciência e Tecnologia do Vale do Paraíba

\section{REFERÊNCIAS}

BARBOSA, R. et al. Produção e sequestro de carbono na atmosfera. Enciclopédia Biosfera, v.9, n.16, p.1783-1798, 2013.

BATISTA, A.F.; BATISTA, G.T. Caracterização fisiográfica e avaliação do uso e ocupação das APP da microbacia do ribeirão das Antas. 2o Seminário de Recursos Hídricos da Bacia Hidrográfica do Paraíba do Sul: Recuperação de Áreas Degradadas, Serviços Ambientais e Sustentabilidade, p.81, 2009.

BRIANEZI, D. et al. Balanço de emissões e remoções de gases de efeito estufa no campus da Universidade Federal de Viçosa. Floresta e Ambiente, v.21, n.2, p.182-191, 2014.

CASTRO, A.G. Estimativa de sequestro de carbono florestal para restauração ecológica devido às emissões de $\mathrm{CO}_{2}$ na instalação de uma Central Geradora Hidrelétrica - CGH. 2017. 143p. (Tese de Doutorado).

DUARTE, L.P.S.S.; MARCHETTO, M. Compensação de $\mathrm{CO}_{2}$ em Eventos. Estudo de Caso: Feira do Empreendedor - MT, Edição 2011. Engineering and Science, v.1, n.1, p.1-12, 2014.

FISCH, G. Caracterização climática e balanço hídrico de Taubaté (S.P). Revista Biociências, v.1, n.1, p.81-90, 1995.

GREENHOUSE GAS PROTOCOL. Metodologia do GHG Protocol da agricultura. 2018. Disponível em: http://www.ghgprotocol. org/sites/default/files/ghgp/standards_supporting/Metodologia .pdf

HIGUCHI, N. et al. Biomassa da parte aérea da vegetação de floresta tropical úmida de terra-firme da Amazônia Brasileira. Acta Amazonica, v.28, n.2, p.153-165, 1998.

IPCC - PAINEL INTERGOVERNAMENTAL PARA AS MUDANCAS CLIMÁTICAS. Historical overview of climate change science, climate change 2007: the physical science basis. 2013. Disponível em: https://www.ipcc-wg1.unibe.ch/publications/ wg1-ar4/ar4-wg1-chapter1.pdf

KRONKA, F.J.N. et al. Monitoramento da vegetação natural e do reflorestamento no Estado de São Paulo. 12 Simpósio Brasileiro de Sensoriamento Remoto, p.1569-1576, 2015.
MARTINS, O.S. Determinação do potencial de sequestro de carbono na recuperação de matas ciliares na região de São Carlos, SP. 2004. 136p. (Tese de Doutorado).

MATTHEWS, H.D. et al. National contributions to observed global warming. Environmental Research Letter, v.9, p.1-9, 2014.

MCT - Ministério da Ciência e Tecnologia. Estimativas anuais de emissões de gases de efeito estufa no Brasil, 2014. Disponível em: http://www.mct.gov.br/upd_blob/0235/235580.pdf.

MELO, A.C.G.; DURIGAN, G. Fixação de carbono em reflorestamentos de matas ciliares no Vale do Paranapanema, SP, Brasil. Scientia Forestalis, v.71, p.149-154, 2006.

OBSERVATÓRIO DO CLIMA. Emissões do Brasil sobem 9\% em 2016. 2017. http://www.observatoriodoclima.eco.br/emissoesbrasil-sobem-9-em-2016/2017

PREISKORN, G.M. Composição Florística, estrutura e quantificação do estoque de carbono em florestas restauradas com idades diferentes. 2011. 129p. (Dissertação de mestrado).

RENNER, R.M. Sequestro de Carbono e viabilização de novos reflorestamentos no Brasil. 2004. 147p. (Dissertação de Mestrado)

RONQUIM, C.C. et al. Carbon sequestration associated to the land-use and land-cover changes in the forestry sector in Southern Brazil. 180 Remote Sensing for Agriculture, Ecosystems, and Hydrology, 2016.

SANQUETTA, C.R. et al. Emissões de dióxido de carbono associadas ao consumo de energia elétrica no Paraná no período de 2010-2014. BIOFIX Scientific Journal, v.2, n.1, p.1-6, 2017.

SANTORO, J.O. et al. Estimativa de recarga do aquífero freático na bacia do Rio Una, no município de Taubaté, SP. Revista Ambiente \& Água, v.2, n.1, p.57-82, 2007.

SÃO PAULO. Inventário florestal da vegetação natural do estado de São Paulo. São Paulo: Editora Páginas e Letras, 2005. Disponível em: http://iflorestal.sp.gov.br/2005/03/01/inventario -florestal-da-vegetacao-natural-do-estado-de-sao-paulo

SIMÕES, S.J.C. Geologia da sub-bacia do Ribeirão das Antas. UNAVALE, 2011. 